\title{
Expectation and Review of Control Strategy of Wind Turbines Yaw System
}

\author{
Jiatong Song \\ School of North China Electric Power University,Baoding,071000,China.
}

249521452@qq.com

Keywords: Wind power generation system,yaw system,yaw control strategy,stable operation.

\begin{abstract}
In recent years China's wind power industry has developed rapidly. Yaw system is an important part of the wind power generation system. Its effectiveness not only affects the wind energy capture efficiency of the system, but also related to the safe operation of the wind power system. In this article, the working principle of the yaw system is described. And the current research status of control mode in China is commented. The function of the yaw system can be divided into two aspects, one is tracing wind accurately, the other is automatic untwisting. Research indicates that, using some new control strategy, such as hill-climbing control(HCC) algorithm, PLC algorithm, fuzzy control, static neural network and many kinds of other algorithm applied to the yaw system, can enhance the stability and robust of yaw system, and increase mechanical life of yaw system. However the technology for automatic untwisting has been ignored, researching new control strategy that can trace wind precisely and achieve automatic twisting is worthy of attention. Determine whether untwisting or not and complete action through the parameters in the algorithm above can improve the economy and stability of yaw system, create great value.
\end{abstract}

\section{Introduction}

Wind power is a clean energy,It has many advantages such as inexhaustible, renewable and environmentally friendly. The utilization of wind energy depends on whether it can accurately trace wind and reduce the loss of wind energy. The yaw control system can adjust wind turbines and make their swept surface vertical to the wind direction, capture wind energy fully and reduce energy loss. Since wind turbines locate in three-dimensional space environment, the uncertainty of wind velocity and direction causes air turbulence, wind shear stress difference and many other factors which can produce large fluctuations during operation, so research on yaw control technology has an important significance for achieving safe and reliable operation of the wind power generation system.

\section{Working principle of yaw system}

Yaw system consists of yaw bearing, yaw driving system, yaw breaks, yaw register, protective device of cable, yaw hydraulic pressure loop and many other parts. There are two main effects, the first is cooperating with the wind turbine control system and tracing the wind accurately, the other is completing the automatic untwisting when the cable twine in engine room and Guarantee the safe operation of the wind turbine.

Yaw system can be classified as passive yaw system and active yaw system. The former is mostly used for small independent wind power generation system, It's controlled by rudder, When the wind direction changes, tracing wind passively. The latter is more applied to large grid-connected wind power generation system. Tracing wind automatically according to the signal delivered by the wind vane downwind. With the wind changing, yaw system requires frequent start to trace wind.

Yaw system works as follows: Wind Sensor working as a sensing element transforms the change of wind into electrical signals and transmits it to the yaw motor loop controller. Yaw control processor issues a command to the yaw motor through comparison and judgment. Yaw motor make the large gear move, the engine room also follows the movement. When the tracing action is 
completed, wind direction and speed sensor lost its signals, yaw motor stop working, then yaw brake locks automatically means yaw process ends.

\section{Research status of yaw control strategy}

China's wind power industry is growing rapidly, and the merits of the strategy yaw control system directly determines the efficiency of the wind turbine. So, advanced yaw system control method and strategy research are the current research focus and hot spot.

\section{1 yaw control system based on PLC governing system}

Xinglin Pei and Mingjie Cheng use AC variable loop governing system to achieve yaw control .The system consists of PLC, inverter and rotary encoder.

In this governing system, the optical rotary encoder that connects with the motor coaxial converting the rotational speed of the motor into voltage $(0-5 \mathrm{~V})$, and feedback it to one input terminal of the PLC analog modules. Then it will be processed with a given amount through PID operation and output a voltage signal(DC $0-10 \mathrm{~V}$ ), the given amount is another analog input signal of analog module which ranges in 0 to $10 \mathrm{~V}$. Finally the output voltage signal controls the inverter output and achieves loop governing control. The goal, precise and steady running speed can be reached .

The system functions above is consistent with the requirement of yaw control system. The actual system is running as follows:

1 The wind direction sensor delivers and transforms wind direction signal to the comparator.

2 The comparator compares wind signal with current direction of engine room and delivers the comparison to the controller.

3 The controller makes mathematical operation to the comparison, the comparison is output to actuator .

4 Actuator begins working, adjust the angle of the engine room, that is to say, yaw operation begins .

5 The result of the yaw operation is feedback to comparator through detecting equipment (Photoelectric encoder or Hall sensor). The above-described process is repeated until the given direction consists with the actual direction of the engine room, yaw control task is completed.

In this system,hardware equipment is: Siemens S7-200 CPU 224X P PLC, Siemens M M 420 inverter, EM 235 analog modules, optical rotary encoder, Three-phase asynchronous AC motors.Xinglin Pei and Mingjie Chen put this technology on wind turbine control laboratory equipment and obtained desired results.

The system has many features. Its structure is simple, configuration is flexible, soft start performance is excellent and its load capacity is great. Its dynamic and steady-state resistance can meet the requirements of the wind turbine yaw control system. According to the control needs, DSC and PC could be connected, HMI monitoring system also could be built. Because of its good stability and accuracy, the system is worthy of study and promotion.

\subsection{Yaw system control strategy based on hill-climbing algorithm}

Qiang Zou, Bofeng Liu et al designed a yaw control system based on hill-climbing algorithm. In order to trace wind accurately, they find the maximum power point. Simulation results verify the accuracy and feasibility. Climbing algorithm begins from the current node, compare it with the neighboring nodes. If the current node is max, then regard it as maximum, otherwise in order to achieve the highest point, replace it with highest neighbor nodes. Yaw system gradually increases the output power by the way of recursive calls. The output power is approaching the maximum output power. In order to achieve a desired value of the maximum output power, we need continuously measure the output voltage and current of generator in suitable time interval. Then wind turbine output power value is obtained. Compare the power value measured with the previous time measured values, use the maximum measurement as new power value. Finally we can get the maximum power value by finding the maximum output power point once and once again. 


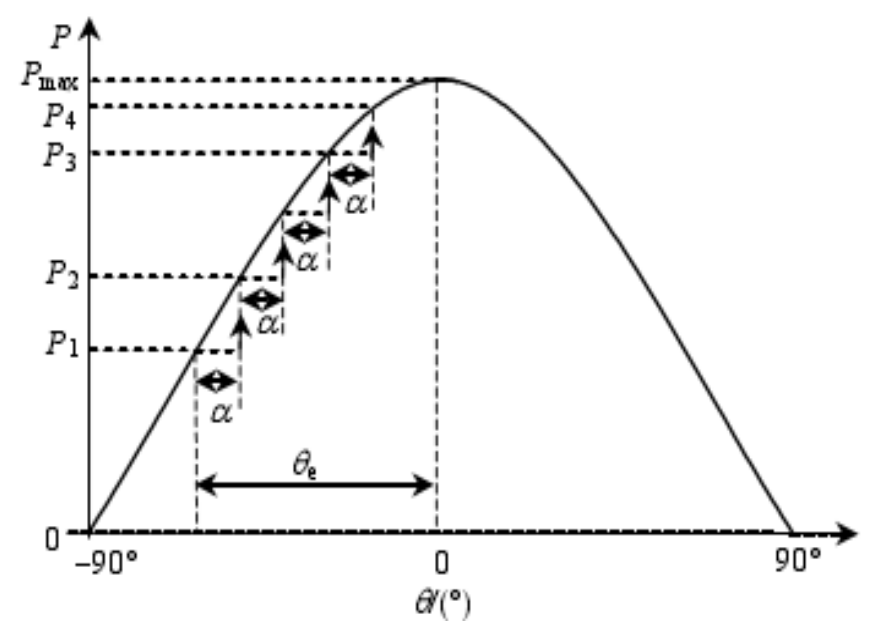

The direction of rotation of the yaw motor is determined by comparison of before and after power value. If the power is bigger, motor Forward. Otherwise Yaw controller controls the motor reversal.

In order to prevent tracing wind frequently, we can set up a threshold to the power. In this threshold, we can consider that trace is accurate and take no measures.

This control strategy has high reliability, its cost is cheaper, and because that wind direction sensors and some other components are eliminating, the loss brought by insufficient accuracy of the sensor itself reduces.

\subsection{Yaw control system based on fuzzy control}

Jianjun Song, Yang Zhang put forward to apply fuzzy control to yaw control system and design a yaw controller and software flow chart of control process. They build a simulation model of the system in Matlab / Simulink Simulation Platform and then do simulation experiment. Comparing the control effect between fuzzy PID and traditional PID, the results show that fuzzy control system can meet the accuracy and stability requirements of yaw control system at the same time. Its overall performance is better than traditional PID control system.

The first step is putting the input and output variables into the fuzzification. Next, create fuzzy control rules. They use MIN-MAX inference method in fuzzy inference of yaw control system and center of gravity method in defuzzification.

\subsection{Yaw control system of PID neural network based on cloud model}

The wind turbine yaw system is a typical no-linear system. It is hard to establish accurate mathematical model. Control performance of the conventional PID control method is difficult to get the desired stability. PID neural network (PIDNN) and classification of the cloud model, wind yaw control. Creating a yaw controller simulation results show that PIDNN system has a high response and steady precision. At the same time, it obtains a better static and dynamic characteristics and robustness.

\section{Discussion and Expectation}

Application of the new control strategy in yaw system greatly improves the energy capture rate, increase the efficiency of the wind turbines. The role of the yaw system has two aspects: First, precisely tracing wind, the second is the automatic untwisting that can protect error happens. However at present, study of Chinese scholars focus only on tracing, automatic untwisting still use the prior technology. The prior technology use gear sensor to determine whether untwist and use cam, worm drive mechanism and a microprocessor to achieve untwisting process. If we can also add automatic untwisting technical into control strategy, determine weather to untwist or not and take action by judging the related parameters, the component of control strategy will be simplified greatly. Such a strategy can meet both economy and safety, its applicability is better. 


\section{Reference}

[1] Xinglin Pei,Mingjie Cheng,et al. The yaw control of PLC governing system in wind turbine[J].Equipment Manufactring Technology. 2011,8:74-76.

[2] Qiang Zou, Bofeng Liu, et al. Application of Hill-Climbing Control Algorithm in Yaw Control System for Wind Power Generation[J].Power System Technology.2010,34(5):72-76.

[3] Jianjun Song, Yang Zhang, et al.Research on the Yaw System of Wind Turbines Based on Fuzzy Control[J].Power System and Clean Energy.2011, 27(7): 78-81.

[4] Haiguo Piao, Zhixin Wang. Simulation of Fuzzy-PID Synthesis Yawing Control System of Wind Turbine[J].Transactions of China electrotechnical society. 2009,24(3):184-188.

[5] Chenghui Zhu, Xiaoya Cheng, et al. Yaw control system of PID neural network based on cloud model[J]. Computer measurement \&control. 2013,21(10), 2700-2703. 